African Crop Science Journal by African Crop Science Society is licensed under a Creative Commons Attribution 3.0 Uganda License. Based on a work at www.ajol.info/ and www.bioline.org.br/cs DOI: https://dx.doi.org/10.4314/acsj.v29i2.3

\title{
NEW SOURCES AND STABILITY OF RESISTANCE TO APHIDS IN COWPEA GERMPLASM ACROSS LOCATIONS IN UGANDA
}

\author{
R. KITYO ${ }^{1}$, J.B. ODOI ${ }^{1}$, A. OZIMATI ${ }^{2}$, I.O. DRAMADRI ${ }^{3}$, R. AGABA ${ }^{1,5}$, P.O. ONGOM ${ }^{6}$, \\ P. NAMPALA ${ }^{4}$, R. EDEMA ${ }^{1,3}$, J. KARUNGI ${ }^{1}$, P. GIBSON ${ }^{3}$ and P.R. RUBAIHAYO ${ }^{1}$ \\ ${ }^{1}$ College of Agricultural and Environmental Sciences, Makerere University, P. O. Box 7062, \\ Kampala, Uganda \\ ${ }^{2}$ National Crops Resources Research Institute, P. O. Box 7084, Kampala, Uganda \\ ${ }^{3}$ Makerere University Regional Centre for Crop Improvement, P. O. Box 7062, Kampala, Uganda \\ ${ }^{4}$ Department of Agriculture and Biological Sciences, Faculty of Science and Technology, Uganda \\ Christian University, P. O. Box 4, Mukono, Uganda \\ ${ }^{5}$ Kachwekano Zonal Agricultural Research and Development Institute, National Agricultural \\ Research Organization, P. O. Box 421, Kabale, Uganda \\ ${ }^{6}$ International Institute of Tropical Agriculture, Nigeria \\ Corresponding author: ronaldkityo@gmail.com
}

(Received 6 December 2020; accepted 30 March 2021)

\begin{abstract}
The cowpea aphid (Aphis craccivora Koch) is an economically important pest, whose feeding effects cause stunting, delayed flower initiation and yield reduction in cowpea (Vigna unguiculata L. Walp). Host plant resistance offers an alternative for controlling aphids; while simultaneously reducing reliance on chemical pesticides. The objective of this study was to evaluate a multi-parent advanced generation inter-cross (MAGIC) population of cowpea against aphids, across cowpea growing regions in Uganda. The study was arranged in alpha lattice design, with two replicates in three locations over two seasons $(2018 \mathrm{~B}$ and 2019A). Results revealed significant effects $(\mathrm{P}<0.001)$ for the main treatment effects, genotype $\mathrm{x}$ location and location $\mathrm{x}$ season interaction for both infestation and damage. The genotype $\mathrm{x}$ season interaction was significant $(\mathrm{P}<0.01)$ for both aphid infestation and damage; while the three-way interaction was only significant $(\mathrm{P}<0.001)$ for aphid infestation, but not for damage. The study identified five new resistant and stable genotypes from the MAGIC panel, including MAGIC131, MAGIC-132, MAGIC149, MAGIC170 and MAGIC280; and one resistant parent, SUVITA-2. The study further revealed MAGIC-125, MAGIC-171, MAGIC153, MAGIC-333, MAGIC177, MAGIC-292, MAGIC282, MAGIC249, MAGIC162, SEC 4W * SEC 5T, NAROCOWPEA4, MAGIC-204, MAGIC039, MAGIC060, MAGIC-097, NAROCOWPEA 3, MAGIC-233, MAGIC090 and MU 9 to be moderately resistant and high yielding genotypes. The above genotypes are recommended for use in the cowpea breeding programme, to develop improved resistant lines against aphids in Uganda.
\end{abstract}

Key Words: Aphis craccivora, MAGIC population, Vigna unguiculata 
Le puceron du niébé (Aphis craccivora Koch) est un ravageur économiquement important, dont les effets alimentaires provoquent un retard de croissance, un retard de floraison et une réduction du rendement du niébé (Vigna unguiculata L. Walp). La résistance des plantes hôtes offre une alternative pour lutter contre les ravageurs destructeurs, y compris les pucerons; tout en réduisant simultanément la dépendance aux pesticides chimiques. L'objectif de cette étude était d'évaluer une population multiparentale inter-croisée de génération avancée (MAGIC) de niébé contre les pucerons, dans les régions de culture du niébé en Ouganda afin d'identifier de nouvelles sources de résistance contre le ravageur. L'étude a été organisée en conception de réseau alpha, avec deux répliques à trois endroits sur deux saisons (2018B et 2019A). Les résultats ont révélé des effets significatifs $(\mathrm{P}<0,001)$ pour les principaux effets du traitement, le génotype x emplacement et l'interaction emplacement x saison pour l'infestation et les dommages. L'interaction génotype $\mathrm{x}$ saison était significative $(\mathrm{P}<0,01)$ à la fois pour l'infestation et les dommages causés par les pucerons; tandis que l'interaction à trois facteurs n'était significative $(\mathrm{P}<0,001)$ que pour l'infestation de pucerons, mais pas pour les dommages. L'étude a identifié cinq nouveaux génotypes résistants et stables du panel MAGIC, notamment MAGIC131, MAGIC-132, MAGIC149, MAGIC170 et MAGIC280; et un parent résistant, SUVITA-2. L'étude a en outre révélé MAGIC-125, MAGIC-171, MAGIC153, MAGIC-333, MAGIC177, MAGIC-292, MAGIC282, MAGIC249, MAGIC162, SEC 4W * SEC 5T, NAROCOWPEA4, MAGIC-204, MAGIC-039, MAGIC060, MAGIC 097, NAROCOWPEA 3, MAGIC-233, MAGIC090 et MU 9 pour être des génotypes modérément résistants et à haut rendement. Les génotypes ci-dessus sont recommandés pour une utilisation dans le programme de sélection du niébé pour développer des lignées améliorées résistantes aux pucerons en Ouganda.

Mots Clés: Aphis craccivora, population MAGIC, Vigna unguiculata

\section{INTRODUCTION}

Cowpea (Vigna unguiculata L. Walp) is one of the most important food grain legumes grown and widely used in the tropics, especially in the semi-arid regions in Africa (Nualsri et al., 2011). It is the fourth most important grain legume in Uganda (Agbahoungba et al., 2017), after beans (Phaseolus vulgaris L.), groundnuts (Arachis hypogaea) and soybean (Glycine max) (Ronner et al., 2012). In Uganda, the crop is mostly grown in the drier eastern and northern parts of the country because of its tolerance to drought and adaptation to warm weather (Dungu et al., 2015). It is also capable of producing reasonable yields, where other grain legumes and cereals fail.

The high contents of carbohydrates $(63 \%)$ and proteins $(25 \%)$ in cowpea grain make it a very important food for human and livestock nutrition (Xiong et al., 2016; Togola et al.,
2020). Despite its importance, cowpea productivity levels are generally low among farmers, ranging between 300 and $500 \mathrm{~kg}$ ha $^{-1}$ (Avosa et al., 2020), compared to its yield potential in the range of 1500 and $3000 \mathrm{~kg}$ ha $^{-1}$ (Gbaye and Holloway, 2011). The crop is heavily infested by cowpea aphid (Aphis craccivora Koch) pest, inflicting damage through direct feeding, and injecting toxic saliva into phloem, leading to stunted growth and sometimes death of the plant during severe infestation (Huynh et al., 2015). Aphid infestation results in reduced photosynthesis (Huynh et al., 2015), delayed initiation of flowering (Souleymane et al., 2013), stunting of plants (Alabi et al., 2012) and yield reduction (Annan et al., 1995). Besides, aphids are the vectors of the two important cowpea diseases in Africa, namely Blackeye Cowpea Mosaic Virus (BCMV) and Cowpea Aphid Borne Mosaic Virus (CABMV) (Boa, 2014). 
In order to control aphids, most farmers in Africa use chemical pesticides (Onyishi et al., 2013). Foliar applications with various insecticides has been reported to be effective against $A$. craccivora (Nualsri et al., 2011); however, rapid development of aphid resistance to pesticides renders chemical treatments ineffective (Karunamoorthi and Sabesan, 2012). Besides, use of chemicals has attracted a lot of public health concerns; apart from contributing to high costs of production (Nicolopoulou-Stamati et al., 2016).

Host plant resistance (HPR) can provide a safe and reliable avenue for aphid pest management, being an environmentally safe and cost-effective pest management technique (Flint et al., 2003). Its potential is generally high because of the availability and access to plant germplasm collections containing genes of resistance; and because of the recent developments in plant science technologies (Ehrhardt, 2014), where plant resistance has been embraced as an important component of integrated pest management.

The most common approaches for evaluating the resistance of cowpea to aphids involve seedling damage, aphid infestation (Omoigui et al., 2017), pod infestation and yield reduction (Zheng et al., 2017). Cowpea germplasm has not been extensively evaluated in Uganda for aphid resistance and this has hindered exploitation of this trait to improve cowpea production in the country. Recently, a multi-parent advanced generation intercross (MAGIC) population was introduced in Uganda, with a view of providing a plant population resource for aphid resistance breeding among other traits.

A MAGIC population typically comprises of 4,8 or 16 founder parents, selected for various desirable traits, such as disease resistance, plant height, flowering time, yield (Riaz et al., 2020) and/or pest resistance. The founder parents are subjected to several generations of intercrossing, followed by multiple generations of selfing to create recombinant inbred lines (RILs), each of which carry a mosaic of the founder haplotypes (Rakshit et al., 2012). Several MAGIC populations have been developed for numerous plant species, including arabidopsis (Kover et al., 2009), wheat (Huang et al., 2012), rice (Bandillo et al., 2013), barley (Sannemann et al., 2015), tomato (Pascual et al., 2015), maize (Jiménez-Galindo et al., 2019) and cowpea (Huynh et al., 2018).

For cowpea, the MAGIC population was created by Huynh et al. (2018), by inter-mating eight parents, using a strategy described in Cavanagh et al. (2008), to produce $315 \mathrm{~F} 8$ recombinant inbred lines of the MAGIC core set. The founder parents used were collected from the Institute of Agricultural Research, Burkina Faso (SUVITA 2); University of California - Riverside, United States (CB27); and; International Institute of Tropical Agriculture, IITA Nigeria (IT93K-503-1, IT89KD-288, IT84S-2049, IT82E-18, IT00K1263 and IT84S-2246). The agronomic and resistance/tolerance characteristics of these parents are described in Huynh et al. (2018). A desirable genotype for improving pest resistance is one which is stable across environments (locations and seasons) (Oliveira et al., 2014). The objective of this study was to identify new sources of resistance to aphids in cowpea collections, through field evaluation across growing locations of cowpea in Uganda.

\section{MATERIALS AND METHODS}

Experimental sites. The study was conducted in three locations in Uganda; namely at (i) Makerere University Agricultural Research Institute, Kabanyolo (MUARIK), located at $0.16^{\circ} 24^{\prime} \mathrm{N}, \quad 325^{\circ} 37^{\circ} \mathrm{E}$, at 1217 meters above sea level (m.a.s.l), with soil being mostly sandy clay loam; (ii) National SemiArid Resources Research Institute (NaSARRI) in Serere district at $1^{\circ} 35^{\prime} \mathrm{N}, 33^{\circ} 35^{\circ} \mathrm{E}$, at 1140 m.a.s.l with black clay soils; and (iii) Ngetta Zonal Agricultural Research and development institute (Ngetta ZARDI) in Lira at $02^{\circ} 17^{\prime} \mathrm{N}$, 
3256 'E, at 1180 m.a.s.l, with sandy loam soils (Otim et al., 2015; Agbahoungba et al., 2017). The experiment was planted in two consecutive rainy seasons in 2018B (August - November 2018) and 2019A (March - June 2019).

Treatments and design. Materials used in the study were obtained from the cowpea gene bank maintained at MUARIK, and contained 279 genotypes including 253 MAGIC lines, 7 founder parents, 4 land races and 15 breeding lines from Uganda. At each of the three experimental sites, the study was established in an alpha lattice design in 14 blocks, each block containing 20 plots (14 blocks x 20 genotypes per block), with two replicates. Each genotype was planted in a two row plot of 8 plants within a row, at a seed rate of two per hill; and later thinned to one plant per hill. The blocks were separated by $1.5 \mathrm{~m}$ alleys with $1 \mathrm{~m}$ between plots. Plant spacing was 75 $\mathrm{cm}$ between and $25 \mathrm{~cm}$ within rows. No pesticides were used and the experiments were planted in isolated fields to avoid effects of pesticide drift from other experimental fields.

Data collection. Six plants were selected from the centre of each row (12 plants selected per plot) for scoring aphid infestation and damage, days to $50 \%$ flowering, days to $50 \%$ physiological maturity (when pod colour was brown/light brown as descried in Cruz et al. (2019)), number of pods per plant, number of grains per pod, 100 grain weight and grain weight per plant per genotype. Aphid infestation and damage symptoms in experimental plots were scored at 30,60 and 80 days after planting (DAP), as recommended by Huynh et al. (2015). Aphid infestation and damage on each of the plants were assessed using a scale of 1 to 5 (Omoigui et al., 2017), as shown in Table 1. Resistance was assessed by scoring for aphid infestation and damage, separately on individual plants.

Data analysis. Data were subjected to analysis of variance (ANOVA), using the Linear Model, with restricted maximum likelihood 
(ReML) statistical procedures in Genstat version 18.0, following the model across locations and seasons as described by Smith et al. (2005) to minimise the sums of squares for the error. The statistical model for ANOVA was:

$$
\begin{aligned}
& y_{i j k l m}=\mu+\rho_{i}+l_{j}+s_{k}+r_{l}+b_{m(l)} \\
& +s_{k j}+\rho s_{k i}+\rho \iota_{j i}+\rho s_{i j k}+\varepsilon_{i j k l m}
\end{aligned}
$$

Where:

$y_{i j k l m}$ is the observed value for the $i^{t h}$ genotype from $j^{\text {th }}$ location, $k^{\text {th }}$ season $m^{\text {th }}$ block nested within the $l^{\text {th }}$ replication;

$i \quad$ is the overall mean;

$\rho_{i} \quad$ is the $i^{t h}$ genotype effect (considered as fixed effect);

$\iota_{j} \quad$ is the $j^{\text {th }}$ location effect (considered as fixed effect);

$s_{k} \quad$ is the $k^{\text {th }}$ season effect (considered as random effect);

$r_{l} \quad$ is the $l^{\text {th }}$ replication effect (considered as random effect);

$b_{m(l)}$ is the effect of $\mathrm{m}^{\text {th }}$ block nested within the $l^{\text {th }}$ replication (considered as random);

$\iota s_{k j} \quad$ is the $k^{\text {th }}$ season and $j^{\text {th }}$ location interaction effect considered as random;

$\rho s_{k i} \quad$ is the interaction effect of $k^{\text {th }}$ season and $i^{\text {th }}$ genotype (considered as random);

$\rho l_{j i} \quad$ is the interaction effect of $j^{\text {th }}$ location and $i^{\text {th }}$ genotype (considered as random);

$\rho l s_{i j k}$ is the effect of the three-way interaction between $k^{\text {th }}$ season, $j^{\text {th }}$ location and $i^{\text {th }}$ genotype (considered as random); and $\varepsilon_{i j k l m}$ is the experimental error considered as random.

Comparative analysis for aphid infestation and damage among genotypes was based on scores captured at 60 DAP, when distinct phenotypic differentiation would be expected among genotypes for the biotic stress factors. Separation of means was done using the ScottKnott test at $5 \%$ level of significance, to allow for the formation of homogeneous groups of treatments and avoid ambiguity in the interpretation of results (Silva, 2007).

Genotype plus genotype by environment (GGE) biplot analysis was performed on resistance (inverse infestation), to explore the genotype plus genotype $\mathrm{x}$ environment interaction. Resistance stability among genotypes was assessed using GGE biplots and Additive Main effects and Multiplicative Interaction (AMMI) scores of the genotypes across environments.

\section{RESULTS}

The results of the combined analysis of variance (ANOVA) for 230 genotypes were used based on completeness of data for aphid infestation, damage and grain yield per plant (Table 2). There were highly significant effects $(\mathrm{P}<0.001)$ for the main treatment effects, genotype by location, and location by season interaction for aphid infestation, damage and grain yield per plant. The genotype by season, and location by season interactions were significant $(\mathrm{P}<0.001)$ for both aphid infestation and damage, but not for grain yield per plant. The three-way interaction was only significant $(\mathrm{P}<0.001)$ for aphid infestation, but not for damage; nor for grain yield per plant (Table 2 ). For aphid damage, highly signification effects were observed for all possible interactions, except for the three-way interaction. Grain yield per plant differed significantly $(\mathrm{P}<0.001)$ among genotypes, locations and seasons. The interaction between 
TABLE 2. Mean squares for aphid infestation, damage and cowpea grain yield per plant in three locations overs two seasons

\begin{tabular}{lrlll}
\hline Source of variation & $\begin{array}{c}\text { Degrees of } \\
\text { freedom }\end{array}$ & \multicolumn{3}{c}{ Mean squares } \\
\cline { 5 - 5 } & & $\begin{array}{c}\text { Aphid } \\
\text { infestation }\end{array}$ & $\begin{array}{c}\text { Aphid } \\
\text { damage score }\end{array}$ & $\begin{array}{c}\text { Grain weight }(\mathrm{g}) \\
\text { per plant }\end{array}$ \\
\hline Rep*(Location*Season) & 6 & $0.75423^{\text {ns }}$ & $3.005^{\text {ns }}$ & $276^{\text {ns }}$ \\
Block*(Rep)(Location*Season) & 156 & $0.23935^{\text {ns }}$ & $0.20124^{\text {ns }}$ & $120.1^{\text {ns }}$ \\
Genotype & 229 & $3.42964^{* * *}$ & $0.84515^{* * *}$ & $1136.5^{* * *}$ \\
Location & 2 & $471.496^{* * *}$ & $523.701^{* * * *}$ & $56898^{* * *}$ \\
Season & 1 & $146.848^{* * *}$ & $1093.55^{* * * *}$ & $15804^{* * *}$ \\
Genotype*Location & 458 & $0.44943^{* * *}$ & $0.53037^{* * * *}$ & $782.6^{* * *}$ \\
Genotype*Season & 229 & $0.46906^{* * * *}$ & $0.34433^{* * * *}$ & $251.7^{\text {ns }}$ \\
Location*Season & 2 & $293.238^{* * *}$ & $8.53395^{* * * *}$ & $2.7^{\text {ns }}$ \\
Genotype*Location*Season & 458 & $0.33297^{* * *}$ & $0.0018^{\text {ns }}$ & $6.9^{\text {ns }}$ \\
Residual & 1218 & 0.34671 & 0.21266 & 232.5 \\
& & & & 408.68 \\
Total & 2759 & 1.25542 & 1.08 & 27.08 \\
S.E.D & & 0.59 & 0.66 & 37.23 \\
C.V & & 25.54 & 23.8 & \\
\hline
\end{tabular}

Note: $* * *$ and $* * *=$ significant at $\mathrm{P}<0.05, \mathrm{P}<0.01, \mathrm{P}<0.001$, respectively; ns = non-significant

genotype and location was also significant $(\mathrm{P}<0.01)$ for grain yield per plant.

Relationships between aphid infestation and cowpea yield traits. The correlation coefficients among the captured resistance to aphids and yield-related traits are shown in Table 3. Aphid infestation and damage were positively correlated $(\mathrm{r}=0.245, \mathrm{P}<0.001)$; while days to $50 \%$ flowering and $50 \%$ maturity correlated positively with aphid damage $(\mathrm{r}=$ 0.486 and $r=0.573$, respectively). Aphid infestation negatively correlated with number of grains per pod, and there was a highly positive correlation $(\mathrm{r}=0.856, \mathrm{P}<0.001)$ between days to $50 \%$ flowering and days to $50 \%$ maturity. Number of pods per plant also correlated positively with grain weight per plant ( $r=0.928)$.

Based on aphid infestation levels, 6 genotypes were found to be resistant (Mean score = 1), 219 moderately resistant (score = 2-3), 4 susceptible (score $=4$ ) and 1 highly susceptible ( score $=5$ ) from evaluations across the three locations (Table 4).

According to infestation scores, genotypes MAGIC131, MAGIC-132, MAGIC280, MAGIC149, MAGIC170 and SUVITA-2 were recorded as resistant (Table 5). Accessions MAGIC301, SECOW 1T, MAGIC-194 and IT93K-503-1 were susceptible; while accession IT82E-18 was the most susceptible (highly susceptible). The rest of the accessions were moderately resistant. None of the local materials evaluated was resistant; while 14 of them were moderately resistant. The best 20 and worst 20 genotypes for aphid resistance based on average aphid infestation scores are presented in Table 5.

Resistance stability of genotypes across locations. Genotype plus genotype by environment (GGE) biplot (Fig. 1) performed on aphid resistance (based on "inverse infestation") explained $90.48 \%$ of the total $\mathrm{G}+\mathrm{GE}$ and revealed that the first principal 
Sources and stability of resistance to aphids in cowpea germplasm

component (PC1) accounted for $67.38 \%$; while the second principal component (PC2) was responsible for $23.10 \%$ of the variation in resistance to aphids. The which-won-where pattern visualisation in the GGE biplot, revealed that a polygon was formed by genotype connectors that were furthest away from the origin (Fig. 1). A polygon view of GGE biplot was formed by connecting the vertex genotypes with straight lines and the rest of the genotypes were placed within the polygon. The vertex genotypes, MAGIC-292, MAGIC006, MAGIC-279 and MAGIC-175, having the longest distances from the origin were more responsive to environmental changes and highly variable in resistance to aphids. The perpendicular lines to the sides of the polygon separated mega-environments. Figure 1 further reveals that the GGE biplot formed three mega-environments, including MUARIK, NaSARRI and Ngetta ZARDI.

Since the analysis contained a large number of genotypes which could not be clearly visualised on the biplots, further analysis was done to explore best genotypes in different locations, using the Additive Main effects and Multiplicative Interaction (AMMI) analysis, and the genotypes ranked according to AMMI resistance stability estimates. In this case, a desirable genotype is one with a high stability estimate, which is associated with resistance performance. Accordingly, the best genotypes for resistance to aphids across all locations based on AMMI averages were MAGIC131, MAGIC-132, MAGIC280, MAGIC149, MAGIC170, MAGIC101, MAGIC-287, MAGIC-139 and SUVITA-2. The best 20 resistant and stable genotypes in each location and across all locations are summarised in Table 6.

Results of the AMMI biplot analysis for genotypes across environments are presented in Figure 2. The biplot showed that the three tested environments were scattered without definite grouping, being consistent with the observation of mega environments in the GGE biplot in Figure 1. Most of the genotypes were clustered close to the midpoint. AMMI biplot 
TABLE 4. Distribution of cowpea genotypes for resistance against cowpea aphids in the study locations in Uganda

\begin{tabular}{|c|c|c|c|c|c|}
\hline \multirow[t]{2}{*}{ Description of infestation } & \multirow{2}{*}{$\begin{array}{l}\text { Aphid } \\
\text { infestation } \\
\text { score }\end{array}$} & \multicolumn{4}{|c|}{ Location } \\
\hline & & Overall & MUARIK & NaSARRI & $\begin{array}{l}\text { Ngetta } \\
\text { ZARDI }\end{array}$ \\
\hline Resistant & 1 & 6 & 102 & 6 & 140 \\
\hline Moderately resistant & $2-3$ & 219 & 123 & 158 & 87 \\
\hline Susceptible & 4 & 4 & 4 & 60 & 2 \\
\hline Highly susceptible & 5 & 1 & 1 & 6 & 1 \\
\hline Total & & 230 & 230 & 230 & 230 \\
\hline
\end{tabular}

MUARIK = Makerere University Agricultural Research Institute, Kabanyolo, NaSARRI = National Semi-Arid Resources Research Institute

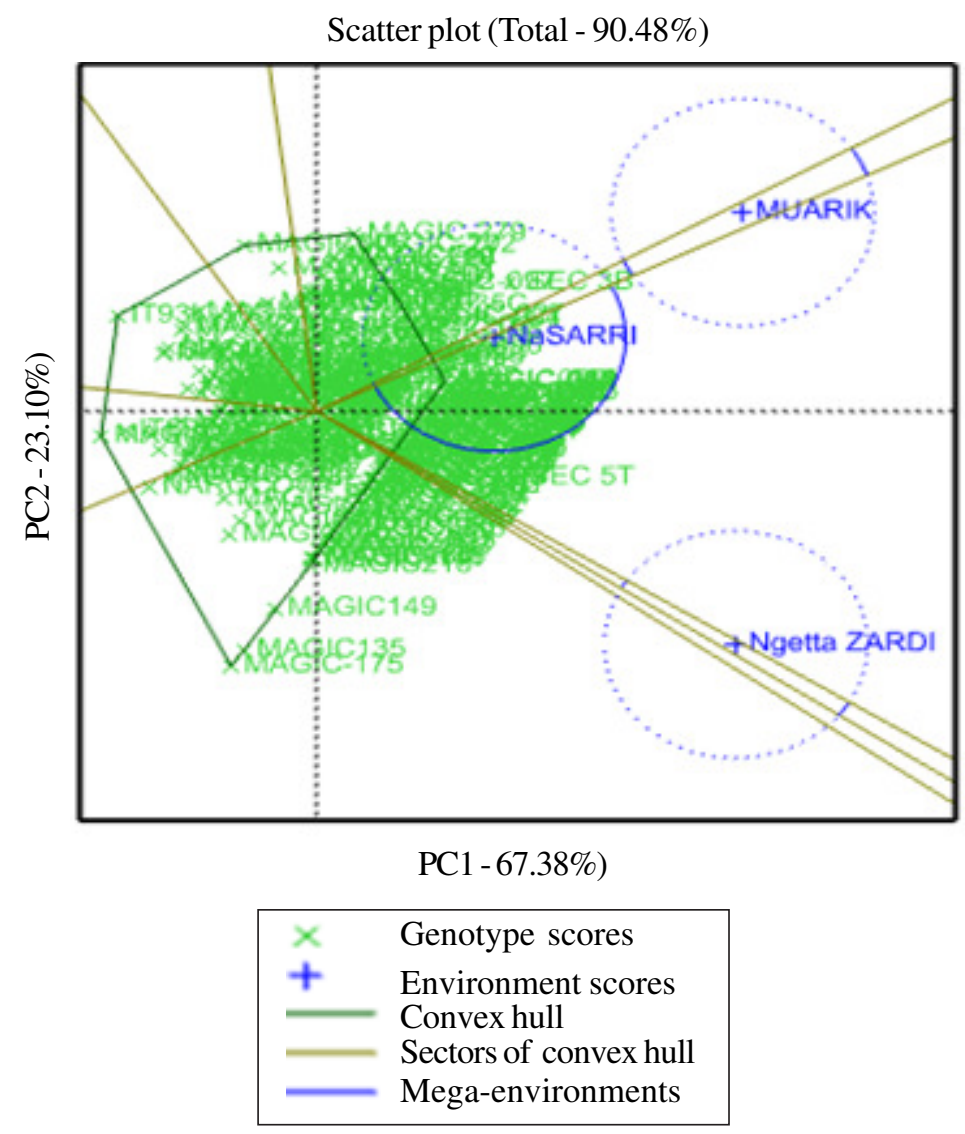

Figure 1. GGE biplot analysis for resistance to aphids among cowpea genotypes across three environments in Uganda. 
TABLE 5. Top 20 most resistant and the 20 most susceptible cowpea genotypes across three environments (locations) based on mean aphid infestation in Uganda

\begin{tabular}{|c|c|c|c|c|c|c|c|c|c|c|c|}
\hline \multicolumn{3}{|c|}{ Overall } & \multicolumn{3}{|c|}{ MUARIK } & \multicolumn{3}{|c|}{ NaSARRI } & \multicolumn{3}{|c|}{ Ngetta ZARDI } \\
\hline Genotype & $\begin{array}{c}\text { Infestation } \\
\quad(\mathrm{x}=2.3 \\
\mathrm{s} . \mathrm{e}=1.19)\end{array}$ & $\begin{array}{c}\text { Resistance } \\
\text { status }\end{array}$ & Genotype & $\begin{array}{c}\text { Infestation } \\
\quad(\mathrm{x}=1.9 \\
\text { s.e }=0.92)\end{array}$ & $\begin{array}{c}\text { Resistance } \\
\text { status }\end{array}$ & Genotype & $\begin{array}{c}\text { Infestation } \\
\quad(x=3.2 \\
\text { s.e }=1.25)\end{array}$ & $\begin{array}{l}\text { Resistance } \\
\text { status }\end{array}$ & Genotype & $\begin{array}{c}\text { Infestation } \\
\quad(\mathrm{x}=1.8 \\
\text { s.e }=0.80)\end{array}$ & $\begin{array}{c}\text { Resistance } \\
\text { status }\end{array}$ \\
\hline MAGIC280 & 1.1 & $\mathrm{R}$ & MAGIC280 & 1.0 & $\mathrm{R}$ & MAGIC131 & 1.3 & $\mathrm{R}$ & MAGIC009 & 1.3 & $\mathrm{R}$ \\
\hline MAGIC131 & 1.1 & $\mathrm{R}$ & MAGIC131 & 1.0 & $\mathrm{R}$ & MAGIC-132 & 1.3 & $\mathrm{R}$ & MAGIC020 & 1.3 & $\mathrm{R}$ \\
\hline MAGIC-132 & 1.1 & $\mathrm{R}$ & MAGIC-132 & 1.0 & $\mathrm{R}$ & MAGIC135 & 1.3 & $\mathrm{R}$ & MAGIC033 & 1.3 & $\mathrm{R}$ \\
\hline MAGIC149 & 1.3 & $\mathrm{R}$ & MAGIC216 & 1.3 & $\mathrm{R}$ & MAGIC149 & 1.3 & $\mathrm{R}$ & MAGIC099 & 1.3 & $\mathrm{R}$ \\
\hline MAGIC170 & 1.3 & $\mathrm{R}$ & MAGIC006 & 1.3 & $\mathrm{R}$ & MAGIC170 & 1.3 & $\mathrm{R}$ & MAGIC101 & 1.3 & $\mathrm{R}$ \\
\hline SUVITA-2 & 1.5 & $\mathrm{R}$ & MAGIC030 & 1.3 & $\mathrm{R}$ & MAGIC280 & 1.3 & $\mathrm{R}$ & MAGIC113 & 1.3 & $\mathrm{R}$ \\
\hline MAGIC101 & 1.6 & MR & MAGIC-035 & 1.3 & $\mathrm{R}$ & MAGIC-078 & 2.0 & MR & MAGIC-122 & 1.3 & $\mathrm{R}$ \\
\hline MAGIC-139 & 1.6 & MR & MAGIC045 & 1.3 & $\mathrm{R}$ & SUVITA-2 & 2.0 & MR & MAGIC-126 & 1.3 & $\mathrm{R}$ \\
\hline MAGIC-287 & 1.6 & MR & MAGIC-046 & 1.3 & $\mathrm{R}$ & MAGIC-287 & 2.2 & MR & MAGIC-139 & 1.3 & $\mathrm{R}$ \\
\hline MAGIC216 & 1.6 & MR & MAGIC-073 & 1.3 & $\mathrm{R}$ & MAGIC-083 & 2.2 & MR & MAGIC157 & 1.3 & $\mathrm{R}$ \\
\hline MAGIC-126 & 1.7 & MR & MAGIC101 & 1.3 & $\mathrm{R}$ & MAGIC101 & 2.2 & MR & MAGIC-171 & 1.3 & $\mathrm{R}$ \\
\hline MAGIC-211 & 1.7 & MR & MAGIC-155 & 1.3 & $\mathrm{R}$ & MAGIC-139 & 2.2 & MR & MAGIC-195 & 1.3 & $\mathrm{R}$ \\
\hline WC 35C & 1.7 & MR & MAGIC-174 & 1.3 & $\mathrm{R}$ & MAGIC-224 & 2.2 & MR & MAGIC-202 & 1.3 & $\mathrm{R}$ \\
\hline MAGIC138 & 1.7 & MR & MAGIC184 & 1.3 & $\mathrm{R}$ & MAGIC276 & 2.2 & MR & MAGIC-211 & 1.3 & $\mathrm{R}$ \\
\hline MAGIC270 & 1.7 & MR & MAGIC-209 & 1.3 & $\mathrm{R}$ & MAGIC-296 & 2.2 & MR & MAGIC242 & 1.3 & $\mathrm{R}$ \\
\hline MAGIC-315 & 1.7 & MR & MAGIC-287 & 1.3 & $\mathrm{R}$ & MAGIC216 & 2.3 & MR & MAGIC-271 & 1.3 & $\mathrm{R}$ \\
\hline MAGIC030 & 1.7 & MR & MAGIC302 & 1.3 & $\mathrm{R}$ & MAGIC-195 & 2.5 & MR & MAGIC280 & 1.3 & $\mathrm{R}$ \\
\hline MAGIC112 & 1.7 & MR & MAGIC-315 & 1.3 & $\mathrm{R}$ & WC 35C & 2.6 & MR & ITOOK-1263 & 1.3 & $\mathrm{R}$ \\
\hline MAGIC-019 & 1.7 & MR & ITOOK-1263 & 1.3 & $\mathrm{R}$ & MAGIC270 & 2.6 & MR & MAGIC-005 & 1.3 & $\mathrm{R}$ \\
\hline MAGIC029 & 1.7 & MR & MAGIC009 & 1.3 & $\mathrm{R}$ & MAGIC112 & 2.6 & MR & MAGIC008 & 1.3 & $\mathrm{R}$ \\
\hline MAGIC-081 & 3.2 & MR & MAGIC300 & 2.9 & MR & MAGIC232 & 4.1 & $\mathrm{~S}$ & MAGIC-129 & 2.6 & MR \\
\hline MAGIC243 & 3.3 & MR & MAGIC-081 & 3.0 & MR & MAGIC-257 & 4.1 & S & MAGIC225 & 2.6 & MR \\
\hline MAGIC176 & 3.3 & MR & MAGIC177 & 3.0 & MR & MAGIC336 & 4.1 & S & MAGIC-292 & 2.6 & MR \\
\hline MAGIC182 & 3.3 & MR & IT93K-503-1 & 3.2 & MR & MAGIC-238 & 4.2 & S & MAGIC176 & 2.8 & MR \\
\hline MAGIC-117 & 3.3 & MR & MAGIC095 & 3.2 & MR & MAGIC297 & 4.2 & S & MAGIC-117 & 2.9 & MR \\
\hline MAGIC-104 & 3.3 & MR & MAGIC-129 & 3.2 & MR & MU 9 & 4.2 & S & MAGIC090 & 3.3 & MR \\
\hline MAGIC-238 & 3.3 & MR & MAGIC-324 & 3.2 & MR & SECOW $1 \mathrm{~T}$ & 4.2 & S & MAGIC-104 & 3.3 & MR \\
\hline MAGIC177 & 3.4 & MR & MAGIC-117 & 3.3 & MR & MAGIC130 & 4.3 & $S$ & MAGIC-107 & 3.3 & MR \\
\hline
\end{tabular}




\begin{tabular}{|c|c|c|c|c|c|c|c|c|c|c|c|}
\hline \multicolumn{3}{|c|}{ Overall } & \multicolumn{3}{|c|}{ MUARIK } & \multicolumn{3}{|c|}{ NaSARRI } & \multicolumn{3}{|c|}{ Ngetta ZARDI } \\
\hline Genotype & $\begin{array}{l}\text { Infestation } \\
\quad(\mathrm{x}=2.3 \\
\mathrm{s} . \mathrm{e}=1.19)\end{array}$ & $\begin{array}{l}\text { Resistance } \\
\text { status }\end{array}$ & Genotype & $\begin{array}{c}\text { Infestation } \\
\quad(\mathrm{x}=1.9 \\
\mathrm{s} . \mathrm{e}=0.92)\end{array}$ & $\begin{array}{l}\text { Resistance } \\
\text { status }\end{array}$ & $\begin{array}{r}\text { Infes } \\
(\mathrm{x} \\
\mathrm{s.e}\end{array}$ & $\begin{array}{l}\text { tation } \\
=3.2, \\
=1.25)\end{array}$ & $\begin{array}{l}\text { Resistance } \\
\text { status }\end{array}$ & Genotype & $\begin{array}{l}\text { Infestation } \\
\quad(\mathrm{x}=1.8 \\
\mathrm{s} . \mathrm{e}=0.80)\end{array}$ & $\begin{array}{l}\text { Resistance } \\
\text { status }\end{array}$ \\
\hline MAGIC-116 & 3.4 & MR & MAGIC166 & 3.3 & MR & MAGIC-303 & 4.3 & $\mathrm{~S}$ & MAGIC130 & 3.3 & MR \\
\hline MAGIC-292 & 3.4 & MR & MAGIC-238 & 3.3 & MR & MAGIC146 & 4.5 & $\mathrm{~S}$ & MAGIC-181 & 3.3 & MR \\
\hline MAGIC-233 & 3.4 & MR & MAGIC-273 & 3.3 & MR & MAGIC176 & 4.5 & $\mathrm{~S}$ & MAGIC182 & 3.3 & MR \\
\hline SECOW $2 \mathrm{~W}$ & 3.4 & MR & MAGIC-194 & 3.4 & MR & MAGIC177 & 4.5 & S & MAGIC-257 & 3.3 & MR \\
\hline MAGIC-107 & 3.4 & MR & MAGIC240 & 3.4 & MR & NAROCOWPEA 2 & 4.5 & S & MAGIC-282 & 3.3 & MR \\
\hline MAGIC-257 & 3.4 & MR & MAGIC-323 & 3.4 & MR & SECOW 5T & 4.5 & S & MAGIC301 & 3.3 & MR \\
\hline MAGIC130 & 3.5 & MR & SECOW 1T & 3.5 & MR & MAGIC243 & 4.6 & HS & NAROCOWF & PEA 13.3 & MR \\
\hline MAGIC301 & 3.6 & S & SECOW 2W & 3.8 & $S$ & MAGIC-292 & 5.0 & HS & SECOW 1T & 3.3 & MR \\
\hline SECOW $1 \mathrm{~T}$ & 3.7 & S & MAGIC301 & 3.8 & S & IT82E-18 & 5.0 & HS & MAGIC-194 & 3.4 & MR \\
\hline MAGIC-194 & 3.9 & S & MAGIC135 & 3.8 & S & IT93K-503-1 & 5.0 & HS & MAGIC-233 & 3.9 & $S$ \\
\hline IT93K-503-1 & 4.4 & S & MAGIC-175 & 3.8 & S & MAGIC-116 & 5.0 & HS & IT82E-18 & 4.5 & S \\
\hline IT82E-18 & 4.8 & HS & IT82E-18 & 5.0 & HS & MAGIC-194 & 5.0 & HS & IT93K-503-1 & 4.9 & HS \\
\hline
\end{tabular}

MUARIK = Makerere University Agricultural Research Institute, Kabanyolo, NaSARRI = National Semi-Arid Resources Research Institute, $\mathrm{R}=$ Resistant, MR = Moderately resistant, $\mathrm{S}=$ Susceptible, HS = Highly susceptible 
TABLE 6. Top 20 AMMI selections of cowpea genotypes based on resistance stability estimates across environments in Uganda

\begin{tabular}{|c|c|c|c|c|c|c|c|c|c|c|c|c|c|c|c|}
\hline \multicolumn{4}{|c|}{ Overall } & \multicolumn{4}{|c|}{ MUARIK } & \multicolumn{4}{|c|}{ NaSARRI } & \multicolumn{4}{|c|}{ Ngetta ZARDI } \\
\hline Genotype & $\begin{array}{l}\text { AMMI } \\
\text { estimate }\end{array}$ & $\begin{array}{l}\text { Infes- } \\
\text { tation } \\
(\mathrm{x}=2.3 \text {, } \\
\mathrm{s} . \mathrm{e}=1.19)\end{array}$ & $\begin{array}{c}\text { Resis- } \\
\text { tance } \\
\text { status }\end{array}$ & Genotype & $\begin{array}{l}\text { AMMI } \\
\text { estimate }\end{array}$ & $\begin{array}{l}\text { Infes- } \\
\text { tation } \\
(\mathrm{x}=1.9, \\
\text { s.e }=0.92)\end{array}$ & $\begin{array}{l}\text { Resis- } \\
\text { tance } \\
\text { status }\end{array}$ & Genotype & $\begin{array}{l}\text { AMMI } \\
\text { estimate }\end{array}$ & $\begin{array}{l}\text { Infes- } \\
\text { tation } \\
(\mathrm{x}=3.2, \\
\mathrm{s} . \mathrm{e}=1.25)\end{array}$ & $\begin{array}{l}\text { Resis- } \\
\text { tance } \\
\text { status }\end{array}$ & Genotype & $\begin{array}{l}\text { AMMI } \\
\text { estimate }\end{array}$ & $\begin{array}{c}\text { Infes- } \\
\text { tation } \\
(\mathrm{x}=1.8 \\
\mathrm{s} . \mathrm{e}=0.80)\end{array}$ & $\begin{array}{l}\text { Resis- } \\
\text { tance } \\
\text { status }\end{array}$ \\
\hline MAGIC131 & 0.80 & 1.1 & $\mathrm{R}$ & MAGIC131 & 0.88 & 1.0 & $\mathrm{R}$ & MAGIC131 & 0.77 & 1.3 & $\mathrm{R}$ & MAGIC009 & 0.78 & 1.3 & $\mathrm{R}$ \\
\hline MAGIC-132 & 0.80 & 1.1 & $\mathrm{R}$ & MAGIC-132 & 0.88 & 1.0 & $\mathrm{R}$ & MAGIC-132 & 0.77 & 1.3 & $\mathrm{R}$ & MAGIC020 & 0.78 & 1.3 & $\mathrm{R}$ \\
\hline MAGIC280 & 0.80 & 1.1 & $\mathrm{R}$ & MAGIC280 & 0.87 & 1.0 & $\mathrm{R}$ & MAGIC135 & 0.75 & 1.3 & $\mathrm{R}$ & MAGIC033 & 0.78 & 1.3 & $\mathrm{R}$ \\
\hline MAGIC149 & 0.76 & 1.3 & $\mathrm{R}$ & MAGIC216 & 0.78 & 1.3 & $\mathrm{R}$ & MAGIC149 & 0.75 & 1.3 & $\mathrm{R}$ & MAGIC099 & 0.78 & 1.3 & $\mathrm{R}$ \\
\hline MAGIC170 & 0.76 & 1.3 & $\mathrm{R}$ & MAGIC006 & 0.77 & 1.3 & $\mathrm{R}$ & MAGIC170 & 0.75 & 1.3 & $\mathrm{R}$ & MAGIC101 & 0.78 & 1.3 & $\mathrm{R}$ \\
\hline MAGIC101 & 0.72 & 1.6 & MR & MAGIC030 & 0.77 & 1.3 & $\mathrm{R}$ & MAGIC280 & 0.75 & 1.3 & $\mathrm{R}$ & MAGIC113 & 0.78 & 1.3 & $\mathrm{R}$ \\
\hline MAGIC-287 & 0.71 & 1.6 & MR & MAGIC-035 & 0.77 & 1.3 & $\mathrm{R}$ & MAGIC-287 & 0.60 & 2.2 & MR & MAGIC-122 & 0.78 & 1.3 & $\mathrm{R}$ \\
\hline MAGIC-139 & 0.71 & 1.6 & MR & MAGIC045 & 0.77 & 1.3 & $\mathrm{R}$ & MAGIC-083 & 0.59 & 2.2 & MR & MAGIC-126 & 0.78 & 1.3 & $\mathrm{R}$ \\
\hline SUVITA-2 & 0.70 & 1.5 & $\mathrm{R}$ & MAGIC-046 & 0.77 & 1.3 & $\mathrm{R}$ & MAGIC101 & 0.59 & 2.2 & MR & MAGIC-139 & 0.78 & 1.3 & $\mathrm{R}$ \\
\hline MAGIC216 & 0.70 & 1.6 & MR & MAGIC-073 & 0.77 & 1.3 & $\mathrm{R}$ & MAGIC-139 & 0.59 & 2.2 & MR & MAGIC157 & 0.78 & 1.3 & $\mathrm{R}$ \\
\hline WC $35 \mathrm{C}$ & 0.69 & 1.7 & MR & MAGIC101 & 0.77 & 1.3 & $\mathrm{R}$ & MAGIC-224 & 0.59 & 2.2 & MR & MAGIC-171 & 0.78 & 1.3 & $\mathrm{R}$ \\
\hline MAGIC270 & 0.69 & 1.7 & MR & MAGIC-155 & 0.77 & 1.3 & $\mathrm{R}$ & MAGIC276 & 0.59 & 2.2 & MR & MAGIC-195 & 0.78 & 1.3 & $\mathrm{R}$ \\
\hline MAGIC-126 & 0.69 & 1.7 & MR & MAGIC-174 & 0.77 & 1.3 & $\mathrm{R}$ & MAGIC-296 & 0.59 & 2.2 & MR & MAGIC-202 & 0.78 & 1.3 & $\mathrm{R}$ \\
\hline MAGIC-211 & 0.69 & 1.7 & MR & MAGIC184 & 0.77 & 1.3 & $\mathrm{R}$ & MAGIC-195 & 0.58 & 2.5 & MR & MAGIC-211 & 0.78 & 1.3 & $\mathrm{R}$ \\
\hline MAGIC030 & 0.68 & 1.7 & MR & MAGIC-209 & 0.77 & 1.3 & $\mathrm{R}$ & MAGIC-078 & 0.58 & 2.0 & MR & MAGIC242 & 0.78 & 1.3 & $\mathrm{R}$ \\
\hline MAGIC-019 & 0.68 & 1.7 & MR & MAGIC-287 & 0.77 & 1.3 & $\mathrm{R}$ & SUVITA-2 & 0.58 & 2.0 & MR & MAGIC-271 & 0.78 & 1.3 & $\mathrm{R}$ \\
\hline MAGIC029 & 0.68 & 1.7 & MR & MAGIC302 & 0.77 & 1.3 & $\mathrm{R}$ & MAGIC216 & 0.54 & 2.3 & MR & MAGIC280 & 0.78 & 1.3 & $\mathrm{R}$ \\
\hline MAGIC-050 & 0.68 & 1.7 & MR & MAGIC-315 & 0.77 & 1.3 & $\mathrm{R}$ & WC $35 \mathrm{C}$ & 0.53 & 2.6 & MR & ITOOK-1263 & 0.77 & 1.3 & $\mathrm{R}$ \\
\hline MAGIC-115 & 0.68 & 1.7 & MR & $\mathrm{ACC} 12 \times \mathrm{SEC} 3 \mathrm{~B}$ & 0.77 & 1.3 & $\mathrm{R}$ & MAGIC270 & 0.53 & 2.6 & MR & MAGIC-004 & 0.77 & 1.3 & $\mathrm{R}$ \\
\hline MAGIC-217 & 0.68 & 1.7 & MR & ITOOK-1263 & 0.77 & 1.3 & $\mathrm{R}$ & $\mathrm{ACC} 12 \times \mathrm{SEC} 3 \mathrm{l}$ & 3В 0.51 & 2.6 & MR & MAGIC-005 & 0.77 & 1.3 & $\mathrm{R}$ \\
\hline
\end{tabular}

MUARIK = Makerere University Agricultural Research Institute, Kabanyolo, NaSARRI = National Semi-Arid Resources Research Institute

$$
\text { is- }
$$
¿ 


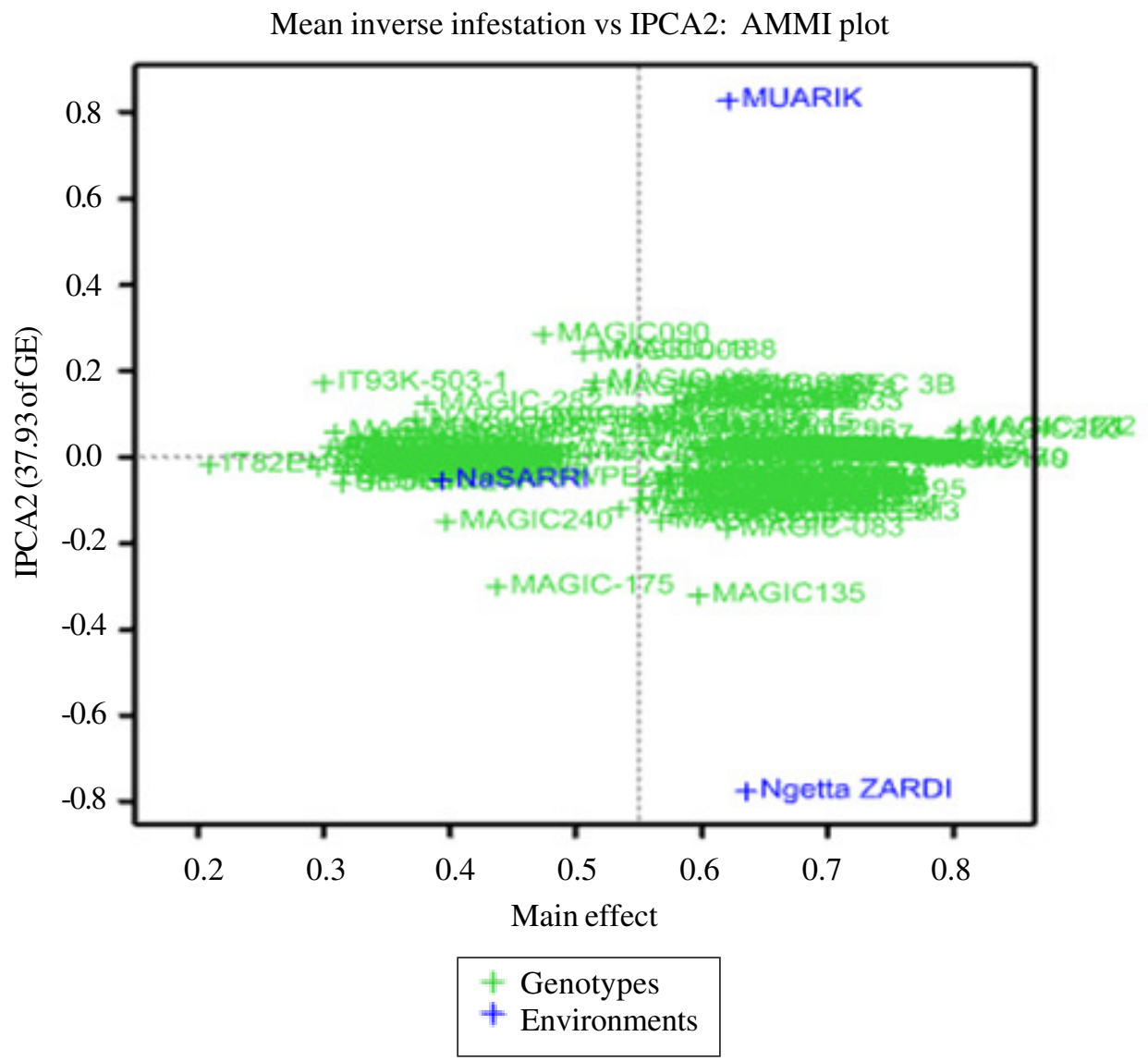

Figure 2. AMMI biplot of IPCA2 scores versus aphid resistance for 230 cowpea genotypes across environments for two seasons (2018B and 2019A) in Uganda.

of IPCA2 scores versus resistance to aphid explained $37.93 \%$ of the total $\mathrm{G} \times \mathrm{E}$ interaction sum of squares.

The results of environment-focused comparison biplot are presented in Figure 3. MUARIK site was located near the centre of the concentric circles as the ideal test location. The other test locations, Ngetta ZARDI and NaSARRI, were less representative.

Grain yield. Genotypes MAGIC-125, MAGIC-171, MAGIC153, MAGIC-333, MAGIC177 and MAGIC-292 had the highest grain weight per plant; all of which were found to be moderately resistant against aphids (Table 7). The best genotypes per location and overall are shown in Table 7. Overall, MUARIK had the highest average grain yield per plant
$(48.2+2.9 \mathrm{~g})$; followed by Ngetta ZARDI $(46.3+1.6 \mathrm{~g})$ and the least was NaSARRI $(25.8+0.8 \mathrm{~g})$. MUARIK and Ngetta ZARDI, which registered the highest grain yield per plant had the lowest aphid infestation scores (scores of 1.9 and 1.8, respectively); and these two locations were not significantly different in both yield and aphid infestation. On the other hand, NaSARRI which registered the lowest grain yield per plant, had the highest aphid infestation score (3.2) and was significantly different from the other two locations based on grain yield and aphid infestation.

\section{DISCUSSION}

The highly significant differences $(\mathrm{P}<0.001)$ among the studied genotypes for cowpea aphid 


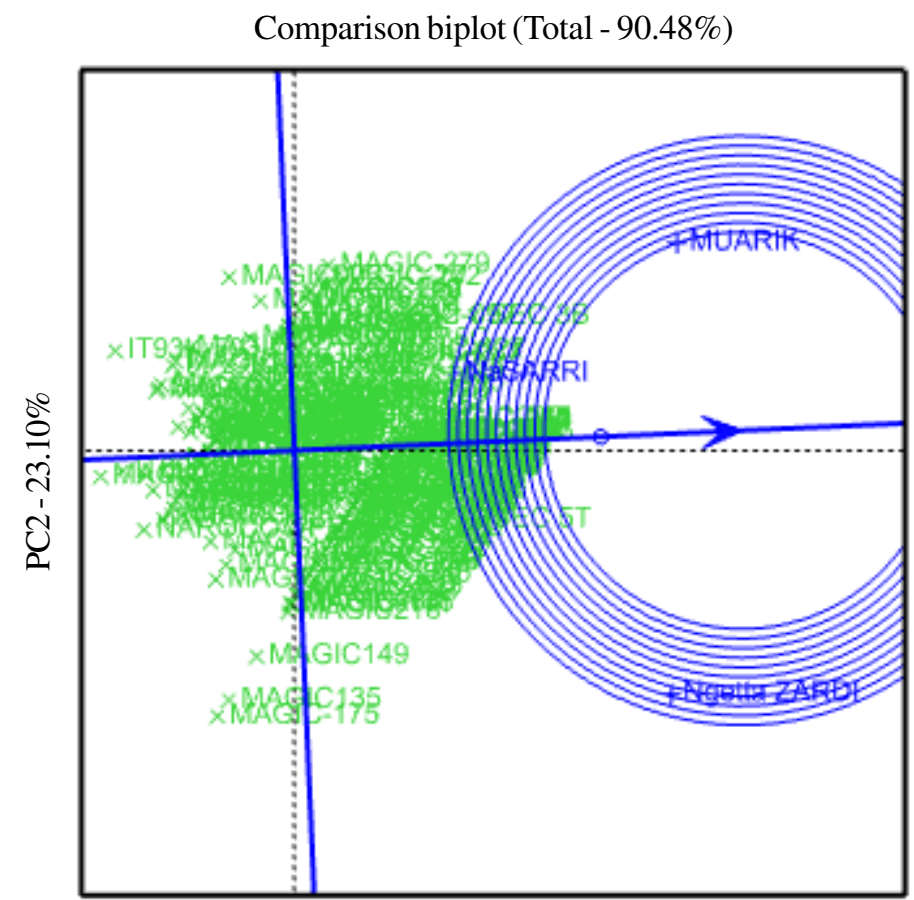

PC1 $-67.38 \%$

$$
\begin{aligned}
& \times \text { Genotype scores } \\
& + \text { Environment scores } \\
& + \text { AEC }
\end{aligned}
$$

Figure 3. Environment-focused comparison biplot for aphid resistance over two seasons in Uganda.

infestation (Table 2), indicates genetic variability among the cowpea materials evaluated; suggesting the possibility of genetically improving cowpea resistance of the locally available cowpea materials against aphids (Avosa et al., 2020).

The significant variations observed among genotypes for aphid resistance could be attributed to biochemical factors affecting behavior and metabolic processes of the aphids; or morphological factors influencing the mechanisms of locomotion, feeding, oviposition, ingestion and digestion of the pest (Onyishi et al., 2013). Togola et al. (2020) argued that aphid resistance in cowpea is associated with low sucrose and high phenolic aglycones (kaempferol and quercetin) content in host plants. It is possible that a combination of biochemical and morphological factors co- influence the resistance trait in cowpea against aphids, and these might be controlled by certain genes (Boukar et al., 2016). Van Emden (1991) earlier reported that resistance to aphids in cowpea is governed by a single dominant gene; further studies to confirm this argument will provide useful information in the improvement of the cowpea resistance against aphid in cowpea in Uganda.

Table 5 shows that the MAGIC accessions MAGIC131, MAGIC-132, MAGIC280, MAGIC149 and MAGIC170 were resistant to aphids. This indicates that the multi-parent advanced generation intercross (MAGIC) population contained sources of resistance against cowpea aphid (Aphis craccivora), which could be used to introgress aphid resistance into farmers' preferred but susceptible cowpea cultivars in Uganda. Since 
TABLE 7. Yield performance of the cowpea genotypes overall and across environments based on AMMI yield values

\begin{tabular}{|c|c|c|c|c|c|c|c|c|c|c|c|}
\hline \multirow[b]{2}{*}{ Genotype } & \multirow[b]{2}{*}{$\begin{array}{l}\text { Resistance } \\
\text { status }\end{array}$} & \multirow[b]{2}{*}{$\begin{array}{l}\text { Grain } \\
\text { weight } \\
\text { per plant } \\
(x=38.46, \\
\text { s.e=29.44) }\end{array}$} & \multicolumn{3}{|c|}{ MUARIK } & \multicolumn{3}{|c|}{ NaSARRI } & \multicolumn{3}{|c|}{ Ngetta ZARDI } \\
\hline & & & Genotype & $\begin{array}{l}\text { Resistance } \\
\text { status }\end{array}$ & $\begin{array}{l}\text { Grain } \\
\text { weight } \\
\text { per plant } \\
(\mathrm{x}=45.28 \\
\mathrm{s} . \mathrm{e}=36.0)\end{array}$ & Genotype & $\begin{array}{l}\text { Resistance } \\
\text { status }\end{array}$ & $\begin{array}{l}\text { Grain } \\
\text { weight } \\
\text { per plant } \\
(x=25.15 \text {, } \\
\text { s.e }=17.0)\end{array}$ & Genotype & $\begin{array}{l}\text { Resistance } \\
\text { status }\end{array}$ & $\begin{array}{c}\text { Grain } \\
\text { weight } \\
\text { per plant } \\
(\mathrm{x}=44.95 \text {, } \\
\text { s.e=27.4) }\end{array}$ \\
\hline MAGIC-125 & MR & 102.39 & MAGIC-125 & $\mathrm{R}$ & 209.46 & MAGIC-292 & HS & 65.06 & MAGIC153 & $\mathrm{R}$ & 195.63 \\
\hline MAGIC-171 & MR & 94.57 & MAGIC-333 & $\mathrm{R}$ & 188.60 & MAGIC-004 & MR & 59.39 & NAROCOWPEA 4 & $4 \mathrm{R}$ & 119.92 \\
\hline MAGIC153 & MR & 86.21 & MAGIC-282 & MR & 169.26 & NAROCOWPEA 1 & $1 \mathrm{~S}$ & 54.80 & MAGIC-094 & MR & 113.58 \\
\hline MAGIC-333 & MR & 83.39 & MAGIC-171 & MR & 154.66 & MAGIC-323 & MR & 54.46 & MAGIC177 & MR & 109.67 \\
\hline MAGIC177 & MR & 79.51 & MAGIC060 & MR & 140.77 & MU 9 & S & 52.15 & MAGIC-262 & $\mathrm{R}$ & 107.73 \\
\hline MAGIC-292 & MR & 78.34 & MAGIC-039 & $\mathrm{R}$ & 136.37 & MAGIC-279 & MR & 51.11 & MAGIC-195 & $\mathrm{R}$ & 105.08 \\
\hline MAGIC-282 & MR & 74.12 & MAGIC184 & $\mathrm{R}$ & 120.68 & MAGIC144 & MR & 48.75 & MAGIC-171 & $\mathrm{R}$ & 101.04 \\
\hline MAGIC249 & MR & 71.96 & MAGIC-238 & MR & 110.69 & ACC12 x SEC 3B & MR & 48.11 & MAGIC249 & $\mathrm{R}$ & 95.22 \\
\hline MAGIC162 & MR & 71.75 & SEC $4 \mathrm{~W} *$ SEC $5 \mathrm{~T}$ & $\Gamma \quad \mathrm{MR}$ & 110.15 & SECOW 5T & $S$ & 47.86 & MAGIC265 & $\mathrm{R}$ & 94.20 \\
\hline SECOW 1T & S & 70.02 & MAGIC-097 & $\mathrm{R}$ & 109.02 & MAGIC-183 & MR & 47.73 & MAGIC-040 & $\mathrm{R}$ & 90.10 \\
\hline SEC 4W * SEC 5T & MR & 69.34 & MAGIC101 & $\mathrm{R}$ & 105.41 & MAGIC-046 & MR & 47.21 & MAGIC033 & $\mathrm{R}$ & 87.10 \\
\hline NAROCOWPEA 4 & MR & 69.15 & MAGIC120 & $\mathrm{R}$ & 104.91 & MAGIC-234 & MR & 47.06 & SECOW 5T & MR & 86.60 \\
\hline MAGIC-204 & MR & 67.25 & MAGIC-204 & MR & 104.24 & MAGIC-181 & MR & 45.85 & MAGIC-129 & MR & 86.38 \\
\hline MAGIC-039 & MR & 66.92 & MAGIC111 & $\mathrm{R}$ & 103.26 & NAROCOWPEA 3 & 3 MR & 44.99 & MAGIC-073 & $\mathrm{R}$ & 86.28 \\
\hline MAGIC060 & MR & 66.87 & SECOW 1T & MR & 101.51 & MAGIC304 & MR & 44.72 & MAGIC090 & MR & 85.68 \\
\hline MAGIC-097 & MR & 66.23 & MAGIC162 & MR & 98.11 & MAGIC006 & MR & 44.68 & MAGIC-152 & $\mathrm{R}$ & 85.15 \\
\hline NAROCOWPEA 3 & MR & 66.12 & MAGIC170 & $\mathrm{R}$ & 97.93 & MAGIC-081 & $\mathrm{S}$ & 43.76 & MAGIC162 & MR & 84.88 \\
\hline MAGIC-233 & MR & 66.06 & SECOW 4W & $\mathrm{R}$ & 95.47 & MAGIC-027 & MR & 43.57 & MAGIC-292 & MR & 84.85 \\
\hline MAGIC090 & MR & 65.26 & MAGIC225 & MR & 95.12 & MAGIC320 & MR & 43.18 & MAGIC-104 & MR & 83.46 \\
\hline MU 9 & MR & 63.67 & MAGIC-087 & $\mathrm{R}$ & 94.02 & MAGIC020 & MR & 42.97 & MAGIC-172 & $\mathrm{R}$ & 81.76 \\
\hline
\end{tabular}

$\mathrm{R}=$ Resistant, $\mathrm{MR}=$ Moderately resistant, $\mathrm{S}=$ Susceptible, $\mathrm{HS}=$ Highly susceptible 
the eight founder parents varied in resistance to aphids, the MAGIC population is likely to segregate for aphid stress resistance traits and could contain lines with unique and novel combinations of resistance genes against aphids. The MAGIC lines generally outperformed the founder parents in their resistance to aphids (Table 6). This could be due to transgressive segregation for the traits in the MAGIC population.

MAGIC populations have great genetic and phenotypic diversity (Huang et al., 2015), providing opportunities for improvement of important traits such as aphid resistance in cowpea, coming from different functional genetic backgrounds. Because of this, the wider phenotypic variations in the MAGIC population could be used to detect quantitative trait loci (QTL) and candidate genes associated with aphid resistance in cowpea.

The founder parent SUVITA-2, which was found to be aphid resistant in this study (Table 5), was also reported to be resistant to Striga, foliar thrips and Macrophomina disease; and tolerant to drought (Hyunh et al., 2018); thus could serve as a source of resistance genes against aphids in Uganda. The founder parents, IT84S-2246 and ITOOK-1263, reported to be resistant to aphids by Huynh $e t$ al. (2018), were moderately resistant in the present study. In South Africa, Letsoalo (2015) reported the cowpea founder parent ITOOK1263 to be resistant against aphids in field experiments; while Onyishi et al. (2013) earlier reported the genotype IT89KD-288 to be resistant against cowpea aphids in Nigeria. These parents were moderately resistant against aphids in Uganda in the present study, indicating the potential of the genotypes in aphid resistance breeding. This discrepancy in their performance might be due to environmental differences in the countries where the other studies were conducted. This underpins the importance of verification trials for the trait of interest in different locations.

The highly significant genotype by environment interaction (GEI) effects observed in the present study (Table 2) indicates that variations among genotypes and environments had pronounced effects on the expression of the resistance traits; suggesting that the cowpea genotypes performed differently in different environments on the resistance trait. This result revealed differential resistance among cowpea genotypes across testing environments, due to the presence of GEI. Since both genotype and environment effects were highly significant $(\mathrm{P}<0.001)$, both factors are important in governing the expression of the aphid resistance traits (Gedif et al., 2014). The presence of GEI could complicate the selection process of superior genotypes, and might reduce the selection efficiency in aphid resistance breeding (Osei et al., 2017). This justifies the exploration of the stability of the resistance and further evaluation of promising genotypes in various test environments. Therefore, widely adapted cowpea genotypes with dynamic resistance stability are recommended to support cowpea production in aphid-prone areas.

The scatter biplot for mega environments delineation and the environment-focused comparison biplot for aphid resistance against aphids in cowpea identified three mega environments with most of the genotypes close to the origin (Fig. 1). This indicates that the tested environments were unique, with specific resistant genotypes; and emphasised the presence of crossover interaction. The top four AMMI selections in Ngetta ZARDI were MAGIC033, MAGIC-126, MAGIC-171 and MAGIC-195. In MUARIK, the top four were MAGIC-132, MAGIC131, MAGIC280 and MAGIC216; while in NaSARRI, the top four were MAGIC-132, MAGIC131, MAGIC149 and MAGIC135 (Table 6). This means that evaluation and recommendation of genotypes based on any single location would be unreliable because of the differences in genetic response across locations (Mare et al., 2017). Under such a situations, it is recommended that genotype evaluation be based on mean performance and stability (Yan and Kang, 
2002). In this case, a desirable genotype is one with a high stability estimate, which is associated with resistance performance (Oliveira et al., 2014).

According to the AMMI stability ranking of genotypes, MAGIC131, MAGIC-132, MAGIC280, MAGIC149, MAGIC170, MAGIC101, MAGIC-287, MAGIC-139 and SUVITA-2 were the most stable in resistance against aphids (Table 6); and are recommended for further investigations. The genotypes appeared to be more resistant in MUARIK than in other test locations (Ngetta ZARDI and NaSARRI). Discriminating and representative test locations are useful for selecting superior genotypes; while eliminating inferior ones (Etnaf et al., 2013). However, since the aphid pressure was highest in NaSARRI; this environment is the best for evaluating promising genotypes for cowpea resistance to aphids.

The positive and significant correlation between aphid infestation and damage indicated linear relationships between these traits (Table 3), suggesting that aphid infestation leads to aphid feeding damage in aphid susceptible genotypes. However, since this correlation coefficient was low, both resistance measures should be considered when screening reaction of cowpea against aphids in field trials. Nevertheless, aphid damage symptoms in field experiments can be confounded by several other factors (such as diseases and other pests) that might be difficult to control leading to misleading results. The positive correlations between days to $50 \%$ flowering and $50 \%$ maturity with aphid damage ( $r=0.486$ and $r=0.573$, respectively) indicate that aphid infestation and damage resulted in delayed flowering and late maturity in susceptible genotypes. Accordingly, the resistant and moderately resistant genotypes showed lower aphid infestation and damage scores, reduced number of days to $50 \%$ flowering; and 50\% maturity and 50\% maturity and higher number of pods per plant.
Souleymane et al. (2013) also reported delayed initiation of flowering in cowpea due to high aphid infestation at vegetative stage; while Zheng et al. (2017) indicated that aphid infestation would result in yield reduction in susceptible cowpea genotypes in the field. This is because aphid infestation reduces the photosynthetic surface due to sooty mould and colony cover, and aphid feeding reduces yield through withdrawing/sucking materials and nutrients that would otherwise be deposited in the sinks for grain filling (Åhman et al., 2019). In the present study, aphid infestation negatively correlated with number of grains per pod, indicating that the infestation caused reduction in number of pods in susceptible genotypes.

\section{CONCLUSION}

The study identified new aphid resistant and stable cowpea genotypes, including MAGIC131, MAGIC-132, MAGIC149, MAGIC170 and MAGIC280 from the MAGIC panel; SUVITA-2 from the parental genotypes; and moderately resistant and stable genotypes MAGIC101, MAGIC-139, MAGIC-287. These genotypes are recommended as promising donor sources for cowpea aphid resistance breeding in Uganda. The moderately resistant genotypes, MAGIC-125, MAGIC171, MAGIC153, MAGIC-333, MAGIC177, MAGIC-292, MAGIC282, MAGIC249, MAGIC162, SEC 4W * SEC 5T, NAROCOWPEA 4, MAGIC-204, MAGIC039, MAGIC060, MAGIC-097, NAROCOWPEA 3, MAGIC-233, MAGIC090 and MU 9, which are also high yielding are recommended for further testing as promising accessions against aphids in cowpea growing areas. The data generated in this study can be used in genome-wide association studies to identify quantitative trait loci (QTL) and candidate genes associated with aphid resistance in cowpea. 


\section{ACKNOWLEDGEMENT}

This research was funded by Carnegie Corporation of New York under the Doctoral Research Grant (RU/2016/Carnegie/DRG/22) and the Post-Doctoral Fellowship Project (RU/ 2020/Post Doc/01) awarded through the Regional Universities Forum for Capacity Building in Agriculture (RUFORUM). Makerere University Regional Centre for Crop Improvement (MaRCCI) provided the germplasm and additional support for field activities.

\section{REFERENCES}

Agbahoungba, S., Karungi, J., Talwana, H., Badji, A., Kumi, F., Mwila, N., Edema, R. Gibson, P. and Rubaihayo, P. 2017. Additive main effects and multiplicative interactions (AMMI) analysis of yield performances in cowpea genotypes under Ugandan environments. International Journal of Advanced Research 5(6):349360.

Åhman, I., Kim, S.Y. and Zhu, L.H. 2019. Plant genes benefitting aphids - potential for exploitation in resistance breeding. Frontiers of Plant Science 10:1452. doi: 10.3389/fpls.2019.01452.

Alabi, O.Y., Aziza, E. and Omoloye, A. A. 2012. Preliminary evaluation of selected cowpea varieties for resistance to cowpea aphid, Aphis craccivora. Nigerian Journal of Ecology 12:45-55.

Annan, I.B., Schaefers, G.A. and Tingey, W.M. 1995. Impact of density of Aphis craccivora Koch. (Aphididae) on growth and yield of susceptible and resistant cowpea cultivars. Annals of Applied Biology 128(2):185-193.

Avosa, O.M., Orawu, M., Ongom, O.P., Dramadri, O.I., Rutayisire, A., Osundwa, C., Gibson, P. and Edema, R. 2020. Establishment of an early selection method (criteria) for breeding in cowpea (Vigna unguiculata). Journal of Plant Breeding and Crop Science 12(2):106-130. doi: 10.5897/JPBCS2019.0843.

Bandillo, N., Raghavan, C., Muyco, P.A., Sevilla, M.A.L., Lobina, I.T., Dilla-Ermita, C.J., Tung, C.-W., McCouch, S., Thomson, M. and Mauleon, R. 2013. Multi-parent advanced generation intercross (MAGIC) populations in rice: Progress and potential for genetics research and breeding. Rice 6(11):1-15.

Boa, E. 2014. Mosaic diseases of cowpea. Africa south health consortium. www.cabi.org.

Boukar, O., Fatokun, C.A., LamHuynh, B., Roberts, P.A. and Close, T.J. 2016. Genomic tools in cowpea breeding programs: Status and perspectives. Frontiers in Plant Science 7:757. doi: 10.3389/fpls.2016.00757.

Cavanagh, C., Morell, M., Mackay, I. and Powell, W. 2008. From mutations to MAGIC: resources for gene discovery, validation and delivery in crop plants. Current Opinion in Plant Biology 11:215221. doi; 10.1016/j.pbi.2008.01.002.

Cruz, J.M.F., Alves, E.U., Farias, O.R., Araújo, P.C. and Oliveira, A.P. 2019. Physiological maturity and determination of the harvest time of Vigna unguiculata L. Walp. Journal of Experimental Agriculture International 34(2):1-8. Article no.JEAI. 48231, doi: 10.9734/JEAI/2019/v34i2 30169.

Dungu, S.P., Ekere, W., Bisikwa, J., Kawooya, R., Kalule, O. and Biruma, M. 2015. Marketing and market integration of cowpea in Uganda. Journal of Development Agricultural Economics 7:111.

Ehrhardt, C., Arapitsas, P., Stefanini, M., Flick, G. and Mattivi, F. 2014. Analysis of the phenolic composition of fungus-resistant grape varieties cultivated in Italy and Germany. Journal of Mass Spectrometry 49:860-869. doi:10.1002/jms.3440.

Flint, M.L., Daar, S. and Molinar, R. 2003. Establishing integrated pest management 
policies and programs: A guide for public agencies.University of California Division of Agriculture and Natural Resources. Publication 8093.

Gbaye, O.A. and Holloway, G.J. 2011. Varietal effects of cowpea, Vigna unguiculata, on tolerance to Malathion in Callosobrachus maculates (Coleoptera: Bruchidae). Journal of Stored Products Research 47:365-371.

Gedif, M., Yigzaw, D. and Tsige, G. 2014. Genotype-environment interaction and correlation of some stability parameters of total starch yield in potato in Amhara region, Ethiopia. Journal of Plant Breeding and Crop Science 6(3): 31-40. doi:10.5897/ JPBCS2013.0426.

Huang, B.E., Verbyla, K.L., Verbyla, A.P., Raghavan, C., Singh, V. K., Gaur, P., Leung, H. Varshney, R.K. and Cavanagh, C.R. 2015. MAGIC populations in crops: Current status and future prospects. Theoretical and Applied Genetics 128:9991017.

Huang, B.E., George, A.W., Forrest, K.L., Kilian, A., Hayden, M.J., Morell, M.K. and Cavanagh, C.R. 2012. A multi-parent advanced generation inter-cross population for genetic analysis in wheat. Plant Biotechnology Journal 10:826-839.

Huynh, B., Ehlers, J.D, Ndeve, A., Wanamaker, S., Lucas, M.R., Timothy, J. Close, T.J. and Roberts, P. A. 2015. Genetic mapping and legume synteny of aphid resistance in African cowpea (Vigna unguiculata L. Walp.) grown in California. Molecular Breeding 35(36):1-9.

Huynh, B., Ehlers, J.D., Munoz-Amatriain, M., Lonardi, S., Santos, J.R.P., Ndeve, A., Batieno, B.J., Boukar, O., Cisse, N., Drabo, I., Fatokun, C., Kusi, F., Agyare, R., Guo, Y., Herniter, I., Lo, S., Wanamaker, S.I., Close, T.J. and Roberts, P.A. 2018. A multi-parent advanced generation inter cross population for genetic analysis of multiple traits in cowpea (Vigna unguiculata $\mathrm{L}$. Walp.). The Plant Journal 93:1129-1142.
Jiménez-Galindo, J.C., Malvar, R.A., Butrón, A., Santiago, R., Samayoa, L.F., Caicedo, M. and Ordás, B. 2019. Mapping of resistance to corn borers in a MAGIC population of maize. BMC Plant Biology 19:431.

Karunamoorthi, K. and Sabesan, S. 2012. Insecticide resistance in insect vectors of disease with special reference to mosquitoes: A potential threat to global public health. Health Scope 2(1): 4-18. doi: 10.17795/jhealthscope-9840.

Kover, P.X., Valdar, W., Trakalo, J., Scarcelli, N., Ehrenreich, I.M., Purugganan, M.D., Durrant, C. and Mott, R. A. 2009. A multiparent advanced generation inter-cross to fine-map quantitative traits in Arabidopsis thaliana. PLoS Genetics 5 e1000551.

Letsoalo, I.M. 2015. Evaluation of introduced cowpea breeding lines for aphid (aphis craccivora) and bruchid (Callosobruchus rhodensiansus) resistance in South Africa Msc. Thesis, University of Limpopo, South Africa. 72pp.

Nicolopoulou-Stamati, P., Maipas, S., Kotampasi, C., Stamatis, P. and Hens, L. 2016. Chemical pesticides and human health: The urgent need for a new concept in agriculture. Frontiers of Public Health 4:148. doi: 10.3389/fpubh.2016. 00148.

Nualsri, C., Potarot, S., Jansod, J., Miloševiæ, D., Wuttiwong, K., Benchasri, S. and Maneelert, V. 2011. Evaluation and utilization of cowpea (Vigna unguiculata (L.) Walp.) germplasm for varietal improvement of resistance to cowpea aphid (Aphis craccivora Koch.) in Thailand. Corpus ID: 53531929.

Oliveira, E.J., Freitas, J.P.X. and Jesus, O.N. 2014. AMMI analysis of the adaptability and yield stability of yellow passion fruit varieties. Scientific Agriculture 71(2):139145.

Omoigui L. O., Ekeuro, G. C., Kamara, A. Y., Bello, L. L., Timko, M. P. and Ogunwolu, G. O. 2017. New sources of aphids [Aphis craccivora (Koch)] resistance in cowpea germplasm using phenotypic and molecular 
marker approaches Euphytica 213:178. doi: 10.1007/s10681-017-1962-9.

Onyishi, G.C., Harriman J. C., Ngwuta, A. A., Okporie, E.O. and Chukwu, S.C. 2013. Efficacy of some cowpea genotypes against major insect pests in southeastern agroecology of Nigeria. Middle-East Journal of Scientific Research 15(1):114-121. doi: 10.5829/idosi.mejsr.2013.15.1.2277.

Osei, M.K., Annor, B., Adjebeng-Danquah, J., Danquah, A., Danquah, E., Blay, E. and Adu-Dapaah, H. 2018. Genotype $\times$ environment interaction: A prerequisite for tomato variety development. http:// dx.doi.org/10.5772/intechopen.76011.

Otim, G.A., Mubiru, D. N., Lwasa, J., Namakula, J., Nanyeenya, W., Okello R. and Elem, J. 2015. Evaluating permanent planting basin for optimum plant populations of maize and beans. Journal of Environmental and Agricultural Sciences 2:2 ISSN: 2313-8629.

Pascual, L., Desplat, N., Huang, B.E., Desgroux, A., Bruguier, L., Bouchet, J.P., Le, Q.H., Chauchard, B., Verschave, P. and Causse, M. 2015. Potential of a tomato MAGIC population to decipher the genetic control of quantitative traits and detect causal variants in the resequencing era. Plant Biotechnology Journal 13:565-577.

Rakshit, S., Rakshit, A., and Patil, J. 2012. Multi-parent intercross populations in analysis of quantitative traits. Journal of Genetics 91:111-117.

Riaz, A., KockAppelgren, P., Hehir, J. G., Kang, J., Meade, F., Cockram, J., Milbourne, D., Spink, J., Mullins, E. and Byrne, S. 2020. Genetic analysis using a multi-parent wheat population identifies novel sources of Septoria Tritici Blotch resistance genes. Genes 11:887. doi: 10.3390/genes 11080887.

Ronner, E. and Giller, K.E. 2012. Background information on agronomy, farming systems and on-going projects on grain legumes in Uganda. pp. 17-18. www.N2Africa.org.
Sannemann, W., Huang, B.E., Mathew, B. and Léon, J. 2015. Multi-parent advanced generation inter-cross in barley: Highresolution quantitative trait locus mapping for flowering time as a proof of concept. Molecular Breeding 35(3):1-16.

Silva, C.M.R. da. 2007. Uso do teste de ScottKnott e da análise de agrupamentos, na obtenção de grupos de locais para experimentos com cana-de-açúcar.. 48p. Dissertação (Mestrado) - Universidade de São Paulo, Piracicaba. doi: 10.11606/ D.11.2008.tde-12032008-151057.

Smith, A.B., Cullis, B.R. and Thompson, R. 2005. The analysis of crop cultivar breeding and evaluation trials: An overview of current mixed model approaches. Journal of Agricultural Science 143(6):449-462.

Souleymane, A., Aken'Ova, M.E., Fatokun, C. A. and Alabi O.Y. 2013. Screening for resistance to cowpea aphid (Aphis craccivora Koch) in wild and cultivated cowpea (Vigna unguiculata L. Walp.) accessions. International Journal of Science, Environment and Technology 2(4): 611-621.

Togola, A., Boukar, O., Servent, A., Chamarthi, S., Tamo, M. and Fatokun, C. 2020. Identification of sources of resistance in cowpea mini core accessions to Aphis craccivora Koch (Homoptera: Aphididae) and their biochemical characterization. Euphytica 216:88, https:/ /doi.org/10.1007/s10681-020-02619-5.

Van Emden, H.F. 1991. The role of host plant resistance in insect pest mismanagement. Bulletin of Entomology Research 81:123126.

Xiong, H., Shi, A., Mou, B., Qin, J., Motes, D., Lu, W., Ma, J., Weng, Y., Yang, W. and $\mathrm{Wu}, \mathrm{D} .22016$. Genetic diversity and population structure of cowpea (Vigna unguiculata L. Walp). PLoS ONE 11(8): e0160941. doi:10.1371/journal. pone. 0160941. 
Zheng, M., Peng, C., Liu, H., Tang, M., Yang, initiation height and branch number in H., Li, X., Liu, J., Sun, X., Wang, X., Xu, J., Hua, W. and Wang, H. 2017. Genomewide association study reveals candidate rapeseed (Brassica napus L.). Frontiers of Plant Science 8:1246. doi: 10.3389/ genes for control of plant height, branch fpls.2017.01246.A. 